\title{
Assembly of specific SR protein complexes on distinct regulatory elements of the Drosophila doublesex splicing enhancer
}

\author{
Kristen W. Lynch and Tom Maniatis \\ Department of Molecular and Cellular Biology, Harvard University, Cambridge, Massachusetts 02138 USA
}

\begin{abstract}
The Drosophila doublesex female-specific splicing enhancer consists of two classes of regulatory elements, six 13-nucleotide repeat sequences, and a single purine-rich element (PRE). Here, we show that the Drosophila regulatory proteins Transformer (Tra) and Transformer 2 (Tra2) recruit different members of the SR family of splicing factors to the repeats and the PRE. The complexes formed on the repeats in HeLa cell extract consist of Tra, Tra2, and the SR protein 9G8. In Drosophila Kc cell extract, Tra and Tra2 recruit the SR protein RBP1 to the repeats. These proteins are arranged in a specific order on the repeats, with the SR protein at the $5^{\prime}$ end of each repeat, and Tra2 at each $3^{\prime}$ end. Although Tra did not cross-link strongly to the repeats, its presence was essential for the binding of Tra2 to the $3^{\prime}$ end of the repeat. Individual SR proteins were also recruited to the PRE by Tra and Tra2, but in this case they were SF2/ASF and dSRp30 in HeLa and Drosophila cell extracts, respectively. The binding of Tra2, Tra, and the specific SR proteins to the repeats or the PRE was highly cooperative within each complex. Thus, Tra2, which contains a single RNA binding domain, can recognize distinct sequences in the repeats and the PRE in conjunction with specific SR proteins. These observations show that the protein composition of each complex is determined by the RNA recognition sequence and specific interactions between SR proteins and Tra and Tra2.
\end{abstract}

[Key Words: Alternative splicing; doublesex; sex determination; SR proteins; transformer proteins; site-specific crosslinking]

Received May 16, 1996; revised version accepted June 26, 1996.

The female-specific alternative splicing of Drosophila doublesex $(d s \mathrm{x})$ pre-mRNA is a well-characterized example of positive control of regulated alternative splicing (for review, see Baker 1989; Steinmann-Zwicky et al. 1990; Maniatis 1991; Rio 1992). The third intron of $d s x$ pre-mRNA contains a nonconsensus $3^{\prime}$ splice site that is not recognized by the splicing machinery in males \{Fig. 1A). Thus, in males the common exons $1-3$ are joined directly to the male-specific exons 5-6. The resulting mRNA encodes a transcriptional regulatory protein required for male sexual differentiation. In contrast, in females the weak $3^{\prime}$ splice site is recognized, resulting in the joining of the common exons 1-3 to the female-specific exon 4. Polyadenylation of this RNA at the end of exon 4 results in an mRNA that encodes a transcriptional regulatory protein required for female sexual differentiation (see Fig. 1A; Burtis and Baker 1989; Coschigano and Wensink 1993).

The female-specific activation of the weak 3 ' splice site requires the proteins Transformer (Tra) and Transformer 2 (Tra2) (McKeown et al. 1988; Nagoshi et al. 1988; Nagoshi and Baker 1990), and a cis-acting regulatory sequence designated the $d s x$ repeat element (dsxRE) (Burtis and Baker 1989; Hedley and Maniatis 1991;
Hoshijima et al. 1991; Ryner and Baker 1991; Tian and Maniatis 1992). Tra is expressed only in females, whereas Tra2 is expressed in both males and females. The $d s x R E$ consists of six 13-nucleotide repeat sequences (Burtis and Baker 1989; Nagoshi and Baker 1990) and a purine-rich element (PRE), both of which are required for efficient use of the female-specific 3' splice site in vitro (Lynch and Maniatis 1995). The $d s x R E$ is capable of activating weak heterologous $3^{\prime}$ splice sites in the presence of Tra and Tra2 and, therefore, is a regulated splicing enhancer (Tian and Maniatis 1992).

Previous studies demonstrated that Tra and Tra2 associate with the $d s x \mathrm{RE}$ as part of a multiprotein enhancer complex (dsxEC), which includes members of the SR protein family of general splicing factors (Tian and Maniatis 1992, 1993). The functional significance of this complex formation was demonstrated by showing that Tra, Tra2, and SR proteins are sufficient to commit $d s x$ pre-mRNA to the splicing pathway in vitro (Tian and Maniatis 1993). A feature shared by all three proteins is the presence of one or more serine-arginine (SR)-rich domains, which are involved in protein-protein interactions and subnuclear localization (Ge et al. 1991; Krainer et al. 1991; Fu and Maniatis 1992; Zahler et al. 1992; Wu 
A

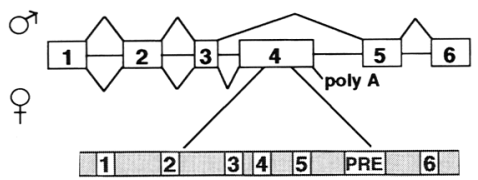

Splicing construct:

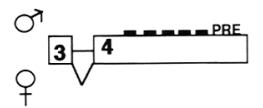

B

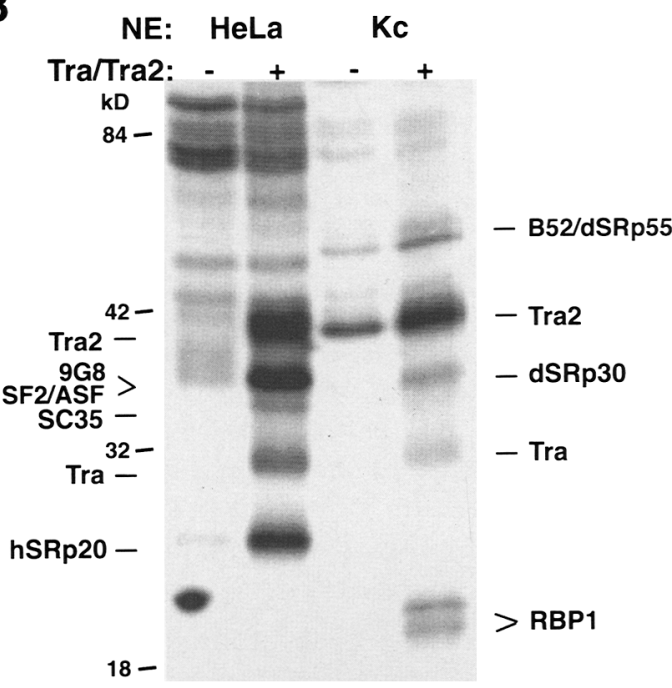

Figure 1. Identification of proteins that cross-link to the $d s x \mathrm{RE}$ under splicing conditions. $(A)$ Schematic of the $d s x$ pre-mRNA, showing the male and female splicing patterns. Open boxes represent exons; lines represent introns. The $d s x \mathrm{RE}$, located within the fourth exon, is shown in detail below the pre-mRNA. Within the $d s x$ RE (shaded box) are labeled the six repeat sequences and the PRE. Also shown is the minigene construct used in splicing assays. This RNA contains only the third exon and intron, and the fourth exon inclusive of repeats $1-5$ and the PRE. $(B)$ Cross-linking of proteins to the $d s x \mathrm{RE}$, in both HeLa and Kc nuclear extracts (NE). Approximate molecular masses are shown under "kD." Reactions labeled + Tra/Tra2 contain $60 \mathrm{ng}$ each of Tra and Tra2. The identified proteins correspond to those that are $\mathrm{Tra} / \mathrm{Tra} 2-$ and $d s \times \mathrm{RE}$-dependent. These proteins are identified in the text.

and Maniatis 1993; Amrein et al. 1994; Kohtz et al. 1994; Hedley et al. 1995; for review, see Fu 1995). In particular, it has been shown that the SR domains within Tra, Tra2, and SR proteins mediate interactions between these factors (Wu and Maniatis 1993; Amrein et al. 1994). In addition, Tra2 and SR proteins, but not Tra, contain a ribonucleoprotein (RNP)-type RNA-binding domain (Boggs et al. 1987; Amrein et al. 1988; for review, see Fu 1995). Thus, the complex formed on the $d s x \mathrm{RE}$ involves both protein-protein and protein-RNA interactions.

Studies with recombinant proteins and with HeLa cell nuclear extract have shown that Tra and Tra2 bind co- operatively, with each other and with SR proteins, to the $d s \times R E$ (Tian and Maniatis 1993, 1994; Lynch and Maniatis 1995). Furthermore, the specificity of the interaction of each of these proteins with the dsxRE is increased dramatically when the proteins are bound in combination rather than in isolation (Lynch and Maniatis 1995). Both the repeat sequences and the PRE have been implicated as potential protein-binding sites within the $d s x \mathrm{RE}$ based on in vitro binding studies with purified recombinant proteins. In addition, functional data indicate that SR proteins may weakly associate with these sequence motifs even in the absence of Tra and Tra2 (Tian and Maniatis 1994; Lynch and Maniatis 1995; Zuo and Maniatis 1996). However, these studies do not address the critical question of where individual components of the $d s x E C$ bind within the $d s x$ RE. Furthermore, although the purification of the $d s x \mathrm{RE}$ showed that SR proteins are the primary components of the $d s x \mathrm{EC}$ along with Tra and Tra2 (Tian and Maniatis 1993), the identification of which SR proteins are bound optimally within the $d s x \mathrm{EC}$ in either human or Drosophila extracts has not been determined.

To address the question of which proteins are bound to the $d s x R E$ as part of the native Tra- and Tra2-dependent $d s x \mathrm{EC}$, and to map the sites where these proteins interact with the $d s x \mathrm{RE}$, we carried out UV cross-linking experiments of enhancer complexes bound to $d s x$ RE RNA. Using both HeLa and Kc cell nuclear extracts, we find that distinct complexes consisting of a specific SR protein, Tra, and Tra2 assemble on each repeat sequence and the PRE in a highly cooperative manner. Remarkably, Tra2 binds specifically to both types of regulatory elements, indicating that the RNA-binding specificity of a protein can be determined by a combination of protein-protein and protein-RNA interactions.

\section{Results}

Identification of $S R$ proteins that are recruited to the dsxRE by Tra and Tra2

Previous studies demonstrated that Tra, Tra2, and a subset of SR proteins are sufficient to commit $d s x$ premRNA to the female-specific splicing pathway in vitro (Tian and Maniatis 1993). Although several different SR proteins can function in this commitment assay, it is possible that particular SR proteins are used preferentially for $d s x$ enhancer-dependent splicing. To address this possibility we carried out UV cross-linking experiments with the $d s x \mathrm{RE}$ assembled in nuclear extracts under conditions similar to those used for splicing. The RNA used in the cross-linking experiments contains the enhancer, but not the intron, to identify only those proteins that bind directly to the $d s x$ RE. Cross-linking assays were carried out by incubating the $d s x R E$ in nuclear extracts, in the presence or absence of Tra and Tra2. The binding reactions also contained excess tRNA and a small amount of BSA to limit nonspecific protein-RNA and protein-protein interactions /see Materials and methods). After binding reached equilibrium (by $20 \mathrm{~min}$; data not shown) proteins were cross-linked to the RNA 
by irradiation with UV light. The RNA was then digested with RNases, such that only proteins that crosslinked at or near a labeled phosphate were linked covalently to a radiolabeled nucleotide. These labeled proteins were then separated on an SDS-PAGE gel and visualized by autoradiography.

The cross-linking experiments were carried out with nuclear extracts from both human HeLa cells and Drosophila Kc cells. The HeLa cell extract allows a direct correlation between cross-linking and splicing activity (see Fig. 4E, below). The Kc extract, although inactive for splicing, identifies the Drosophila proteins that interact specifically with the dsxRE. The results of the crosslinking assay using uniformly labeled $d s x$ RE RNA are shown in Figure 1B. In the absence of Tra and Tra2 only a few slow-migrating proteins appear to cross-link significantly to the RNA in HeLa cell extract. The crosslinking of these proteins to RNA is not dependent on the presence of $d s x$ RE sequences (data not shown). Furthermore, the amount of cross-linking of these proteins frequently decreases in the presence of Tra and Tra2 (see Figs. 2A and 3A). Thus, the bands observed in the absence of Tra and Tra2 represent nonspecific proteinRNA interactions, which likely correspond to hnRNP proteins (Bennett et al. 1992). We also note that the Kc extract appears to contain low levels of endogenous Tra2; however, no endogenous Tra is detected (Fig. 1B).

In contrast, a number of additional proteins cross-link to the $d s x \mathrm{RE}$ in the presence of Tra and Tra2 (Fig. 1B), and this cross-linking requires the $d s \times R E$ (data not shown). Consistent with previous studies (Tian and Ma- niatis 1993), all of these proteins are members of the SR protein family. 9G8 and SF2/ASF /which comigrate in these experiments), SC35, hSRp20, B52/dSRp55, Tra, and $T r a 2$ were all identified based on comigration with recombinant proteins, or comigration with bands detected by Western blot analysis using antibodies against these proteins, or both (data not shown). Cross-linked recombinant proteins migrate exactly with the crosslinked proteins from the extract, whereas Western blots done on cross-linked samples detect both a cross-linked and an uncross-linked population of protein. RBP1 as well as SF2/ASF and Tra2 were identified based on immunoprecipitation of the cross-linked protein with specific antibodies (data not shown; see Fig. 5C, below). The identity of the $35-\mathrm{kD}$ protein in Kc extract (dSRp30) could not be confirmed because no SR proteins of this molecular mass have been cloned from Drosophila. However, purification of total SR proteins from Drosophila does reveal the presence of a protein with molecular mass of $\sim 35 \mathrm{kD}$ (Roth et al. 1991). Because the protein we detect at $35 \mathrm{kD}$ in Kc extract binds to the $d s x \mathrm{RE}$ with specificity similar to that of SF2/ASF (see Fig. 5A,B, below), it is likely to be dSRp30, and we will refer to it as such here.

Tra and Tra2 form a specific complex with 9G8 or $R B P 1$ on the repeat sequences

The cross-linking experiments carried out with uniformly labeled RNA identify all of the proteins that interact with the $d s x$ RE in a Tra- and Tra2-dependent manner, but they do not show where these proteins interact
A

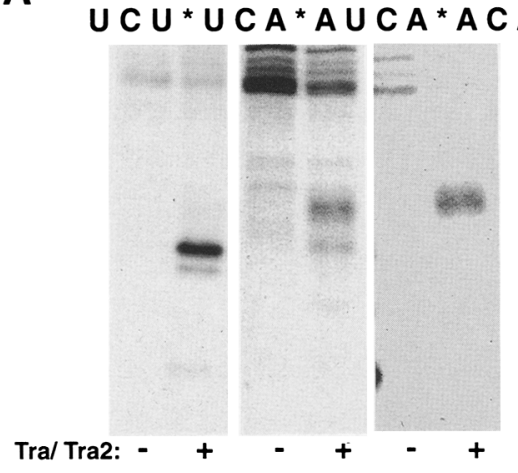

Kc

$U C U$ * $U C A$ * $A U C A * A C A$

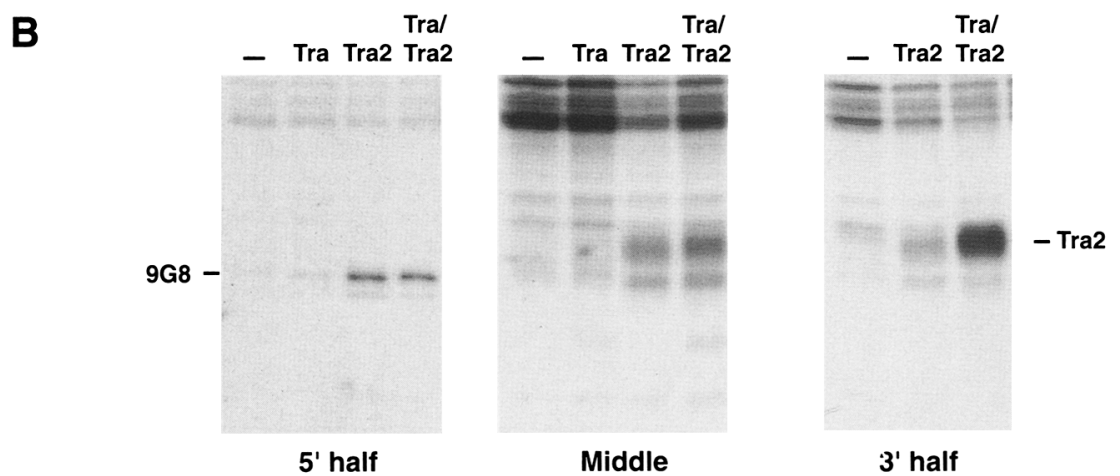

Figure 2. Site-specific cross-linking of proteins to repeat 4 in the context of the intact dsxRE. (A) Cross-linking of proteins in $\mathrm{HeLa}$ and $\mathrm{Kc}$ nuclear extracts to single labels within repeat 4 . Single labels within the repeat 4 sequence are designated *, positioned above the lanes to which they correspond. (B) Cross-linking of proteins in HeLa extract to $5^{\prime}$ half (UCU*UCAAUCAACA), middle (UCUUCA *AUCAACA), or $3^{\prime}$ half (UCUUCAAUCA ${ }^{\star} A C A$ ) of repeat 4 with addition of Tra alone, Tra 2 alone, or Tra and Tra 2 as indicated. 
A
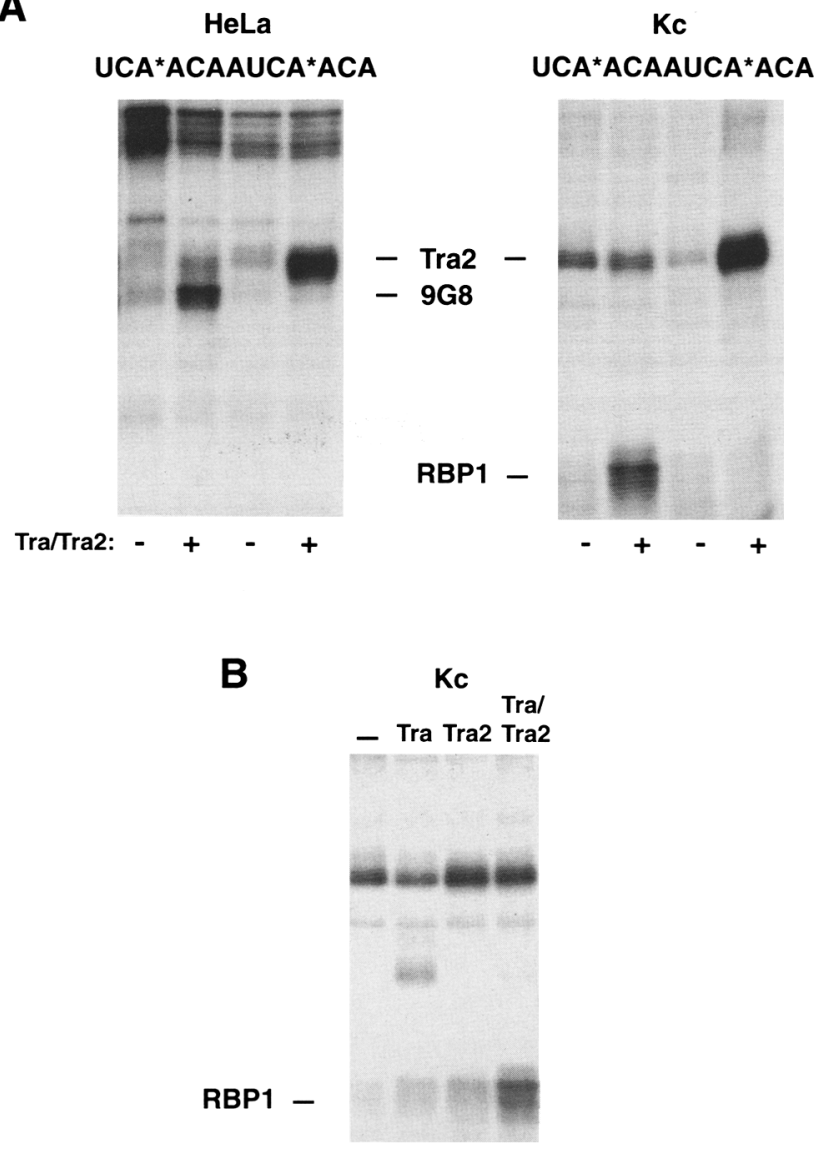

5 ' half

Figure 3. Site-specific cross-linking of proteins to repeat 5 in the context of the intact $d s x$ RE. (A) Cross-linking of proteins in $\mathrm{HeLa}$ and $\mathrm{Kc}$ nuclear extracts to single labels within repeat 5 . Locations of single labels are designated as in Fig. 2A. (B) Crosslinking of proteins in Kc cell extract to the $5^{\prime}$ half $\left(\mathrm{UCA}^{*} \mathrm{AC}\right.$ AAUCAACA) of repeat 5 with addition of Tra alone, Tra 2 alone, or Tra and Tra2.

along the $d s x$ RE. Therefore, we carried out UV crosslinking studies with $d s x$ RE RNA containing a singlelabeled phosphate at a specific position within the repeat element using the site-specific labeling procedure of Moore and Sharp (1992) (see Materials and methods for details).

The $d s x$ RE repeats 1,2 , and 4 are identical, whereas repeats 3,5 , and 6 differ from this consensus sequence by 1,2 , and 3 nucleotides, respectively. Therefore, we first introduced labeled phosphates in the $5^{\prime}$ half, the $3^{\prime}$ half, and the middle of repeat 4 to identify proteins that crosslink to a consensus repeat. These single labels were placed within the full $d s x$ RE RNA, so that we could assay for binding to specific sites within the fully assembled $d s x E C$. As shown in Figure 2A, in HeLa cell extract a $30-\mathrm{kD}$ protein, which we identified as 9G8 (Fig. 4), specifically cross-linked to the $5^{\prime}$ end of the repeat, whereas Tra 2 cross-linked to the $3^{\prime}$ end of the repeat. In Kc extract Tra2 also cross-linked to the $3^{\prime}$ half of the repeat, whereas RBP1 cross-linked to the $5^{\prime}$ half. We did not detect SR proteins at the 3 ' half of the repeat nor significant amounts of $\operatorname{Tra} 2$ at the $5^{\prime}$ half; however, when the single label is placed in the middle of the repeat it cross-links with intermediate intensity to the proteins bound at either end. In addition, very weak crosslinking of Tra to the middle of the repeat was detected in the HeLa cell extract. We also note that many proteins appear to cross-link to the middle of the repeat in the $\mathrm{Kc}$ extract, but they are observed in both the absence and the presence of Tra and Tra2. Because none of these bands correspond to proteins that specifically cross-link as part of the $d s x \mathrm{EC}$ (Fig. 1B), they likely represent nonspecific binding.

To determine the relative roles of Tra and Tra2 in promoting specific protein-RNA interactions in the $d s x$ RE, we repeated the cross-linking experiments in the presence of only Tra or Tra2. The addition of Tra2 to HeLa nuclear extract was sufficient to induce the cross-linking of $9 \mathrm{G} 8$ to the $5^{\prime}$ half of repeat 4 , and the further addition of Tra did not affect the strength of this interaction (Fig. 2B). Likewise, Tra2 alone is necessary and sufficient for the cross-linking of 9G8 and Tra2 to the middle of the repeat. Surprisingly, however, the binding of Tra2 to the $3^{\prime}$ half of repeat 4 is almost entirely dependent on the presence of Tra (Fig. 2B), as well as the presence of 9G8 (data not shown). This involvement of Tra in the recognition of the repeat is consistent with the weak association of Tra to the middle of the repeat (see Discussion). Similar results were obtained when Tra and Tra2 were added separately to Drosophila Kc cell extract (data not shown).

Next, we placed site-specific labels in repeat 5 to determine whether its sequence deviation from consensus alters the pattern of protein cross-linking. The $5^{\prime}$ half of repeat 5 contains two adenine residues in place of the two uracils in the consensus repeat sequence (cf. Fig. 2A and $3 \mathrm{~A})$. One effect of these changes is that the resulting repeat 5 sequence consists of two direct repeats of the sequence UCAACA separated by a single adenine. If the RNA sequence is the primary determinant of binding, then it is likely that within the complex binding to repeat 5 a Tra 2 molecule would associate with each half of this repeat, as $\operatorname{Tra} 2$ recognizes the 3' UCAACA sequence in repeat 4 .

Contrary to that prediction, however, when a single label is placed between the two adenines in the $5^{\prime}$ half of repeat 5 , the predominant cross-linked species is again 9G8 in HeLa extract and RBPl in Kc extract (Fig. 3A). There is weak association of Tra2 with this site in both extracts. However, because this association does not increase in Kc extract upon the formation of the $d s \times \mathrm{EC}(+$ Tra and Tra2; Fig. 3A), most likely Tra2 does not interact specifically with the $5^{\prime}$ half of repeat 5 within the $d s x E C$ but rather associates weakly to that site when the $d s \times E C$ has not formed (see also Fig. 4B). Tra2 does, however, cross-link strongly to the $3^{\prime}$ half of repeat 5 . Thus, despite the sequence variations between repeat 4 and repeat 5 , both repeats associate with the same proteins in the same spatial arrangement. Therefore, repeat 5 may 

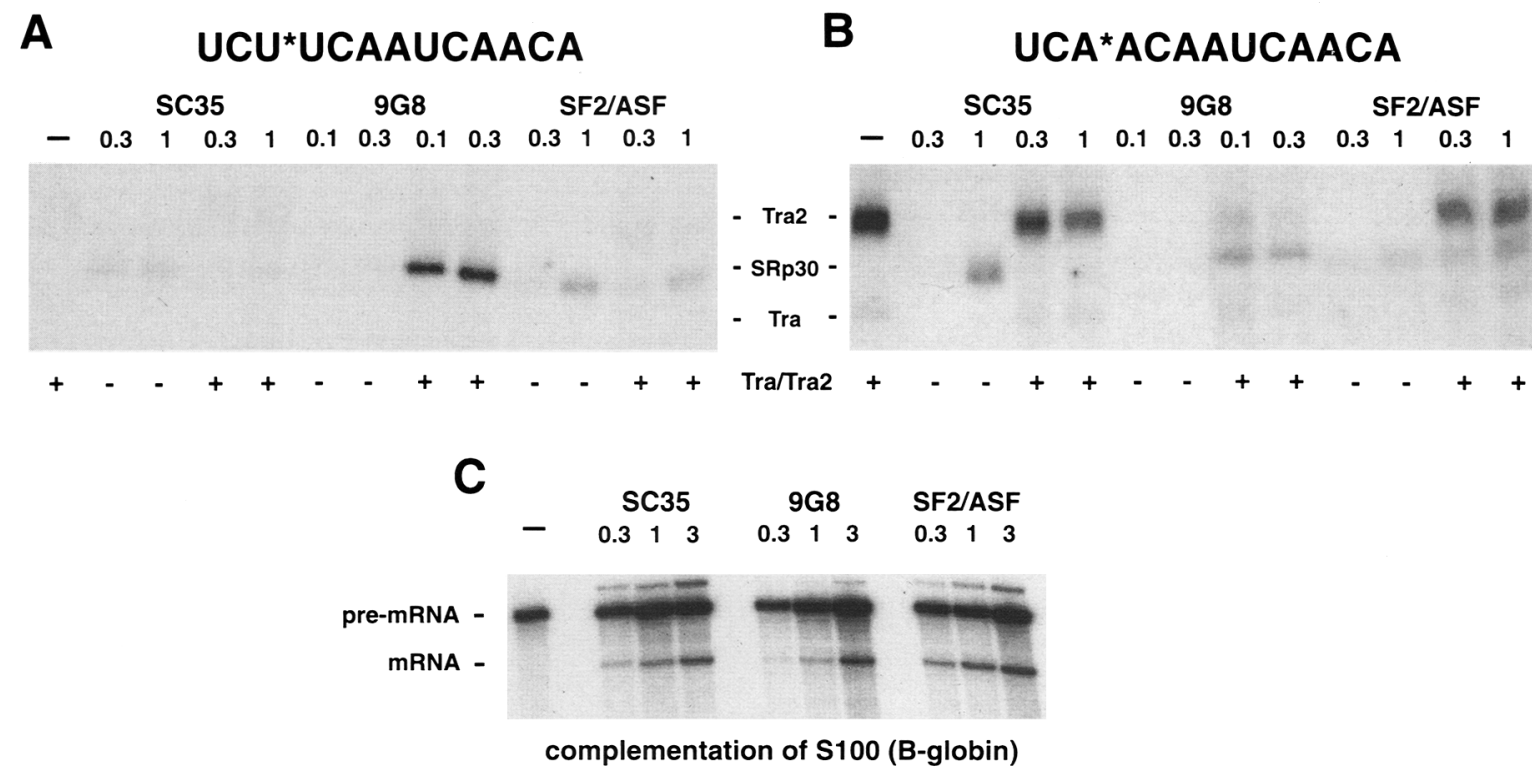

D

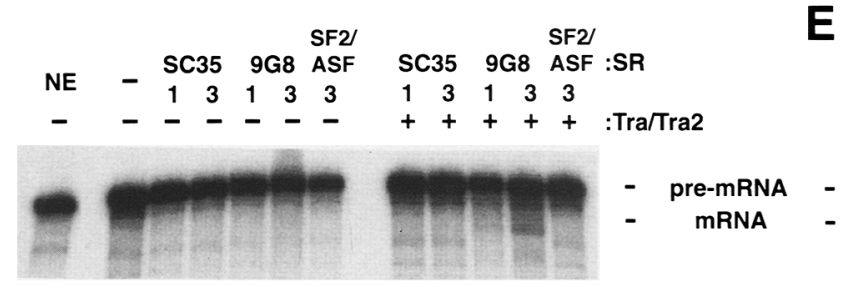

complementation of $\mathrm{S} 100(\mathrm{dsx})$
$\mathbf{E}$

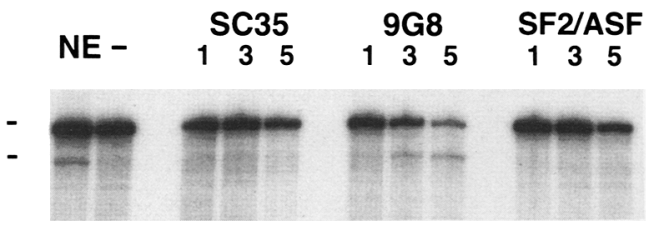

commitment of dsx

Figure 4. 9G8 specifically cross-links to the $5^{\prime}$ half of repeat sequences and is critical for the function of the $d s x E C$. $|A|$ Cross-linking of purified recombinant SC35, 9G8, and SF2/ASF in the presence and absence of Tra and Tra2 to the 5' half of repeat 4 (UCU*UCAAUCAACA). No nuclear extract was present in this experiment. Units of SR proteins are given in microliters. All SR proteins used are present at a concentration of $\sim 100 \mathrm{ng} / \mu \mathrm{l}$. (B) Cross-linking as in $A$ but to the $5^{\prime}$ half of repeat 5 (UCA ${ }^{*}$ ACAAUCAACA). (C) Splicing of $\beta$-globin pre-mRNA in S100 extract complemented with SC35, 9G8 or SF2/ASF. Units of SR proteins are as described in $A$. Correctly spliced product is labeled as mRNA. (D) Splicing of the $d s x$ construct D1 (Tian and Maniatis 1992), which contains repeats 1-5 and the PRE, in S100 extract supplemented with SC35, 9G8, and SF2/ASF in the presence or absence of Tra and Tra2. Correctly spliced product is labeled as mRNA. In reactions that do not splice, a background band migrates just below where the mRNA migrates. $(E) d s x$ commitment complex assay as described in Tian and Maniatis (1993). D1 pre-mRNA is incubated for 1 hr with Tra and Tra2 either alone (-) or in the presence of SC35, 9G8, or SF2/ASF as indicated. Nuclear extract is then added with a 150 -fold excess of $d s x$ RE-specific competitor. Only those reactions in which a stable complex formed on the $d s x$ pre-mRNA during the preincubation are chased into spliced product.

represent a suboptimal repeat sequence. Consistent with this possibility is the observation that although crosslinking of both $9 \mathrm{G} 8$ and RBP1 to the $5^{\prime}$ half of repeat 4 requires only Tra2, as does the association of 9G8 with the $5^{\prime}$ half of repeat 5 , optimal binding of RBP1 to the $5^{\prime}$ half of repeat 5 requires Tra as well as Tra2 (Fig. 3B). Thus, the changes of uracil to adenine within the 5 ' half of repeat 5 appear to decrease the ability of RBP1 to bind to this site, such that interactions with both Tra and Tra2, instead of with Tra2 alone, are required to facilitate the binding of RBP1 to repeat 5 .

The association of $9 G 8$ with the repeat sequences is functionally significant

The binding of RBPl to the repeats in Kc extract was identified on the basis of migration of the cross-linked species and confirmed by immunoprecipitation of RBP1 cross-linked to the $5^{\prime}$ half of repeat 4 (data not shown). In contrast, in HeLa extract the molecular mass of the protein(s) that cross-linked to repeats 4 and 5 was insufficient to identify which of the hSRp30 proteins was binding. The migration suggested that the major component was either 9G8 or SF2/ASF, whereas SC35 bound only very weakly to this site (cf. Figs. $2 A$ and $1 B$ ).

To determine conclusively which hSRp30, if any, was binding cooperatively with Tra and Tra 2 to the repeat sequences, we cross-linked single-labeled RNA in the presence of Tra, Tra2, and purified recombinant SC35, 9G8 la gift from J. Stevenin, Centre National de la Recherche Scientifique (CNRS), Strasbourg, France], or SF2/ASF. As shown in Figure 4A, B, SC35 and SF2/ASF 
cross-link very weakly to the $5^{\prime}$ half of repeats 4 and 5 either with the absence or presence of Tra and Tra2. In striking contrast, 9G8 cross-links strongly to repeats 4 and 5 in the presence, but not in the absence, of Tra and Tra2, at amounts $\geqslant 10$-fold lower than the amount of SC35 or SF2/ASF used. Thus, the ability of recombinant 9G8 to cross-link to the repeats is indistinguishable from the cross-linking of the SRp30 detected in HeLa cell extract, and 9G8 binds to the $5^{\prime}$ half of the repeats with an affinity $>10$-fold more than that of either SC35 or SF2/ ASF. Interestingly, in the absence of 9G8 there is significant cross-linking of Tra2 to the 5' half of repeat 5, but this cross-link is competed away when 9G8 is present (Fig. 4B). This is consistent with the $5^{\prime}$ sequence UCAACA acting as a binding site for Tra2 in the presence of Tra, but preferred by 9G8 when the full complex is formed on the repeat.

Although it was clear that 9G8 binds specifically to the repeats in the presence of Tra and Tra2, previous studies had indicated that SC35 could form a complex with Tra and Tra2 on the $d s x R E$ that committed the substrate to the female pattern of splicing (Tian and Maniatis 1993). Therefore, we wanted to determine whether the association of $9 \mathrm{G} 8$ with the repeats is functionally significant. Recombinant 9G8, SF2/ASF, and SC35 all were equivalently active for the complementation of splicing of a $\beta$-globin transcript in $\mathrm{S} 100$ extract (Fig. 4C). However, only 9G8 was able to stimulate significantly the splicing of the $d s x$ substrate when $\$ 100$ extracts were complemented with Tra, Tra2, and the hSRp30s (Fig. 4D). The activity of 9G8 was also tested using a commitment complex assay (Tian and Maniatis 1993) in which Tra, Tra2, and hSRp30s were preincubated with the $d s x$ substrate followed by the addition of a vast excess of $d s x$ RE RNA competitor and nuclear extract. Consistent with earlier results, preincubation with Tra and Tra2 alone was insufficient to form a stable committed complex. However, addition of $9 \mathrm{G} 8$ to the preincubation with Tra and Tra 2 commits $\$ 50 \%$ of the substrate to the female-specific pattern of splicing (Fig. 4E). The fact that we could not detect any significant effect of SC35 preincubation with Tra and Tra2 in this study is likely attributable to differences in the quantity or activity of the proteins used in this experiment relative to the earlier study (Tian and Maniatis 1993).

In summary, we find that the same SR proteins are recruited to repeats 4 and 5 by Tra and Tra2, and this complex formation occurs on isolated repeat sequences (see Fig. 6, below). Thus, Tra2, Tra, and 9G8 or RBP1 form a heterotrimeric complex (a repeat-specific complex or $d s x \mathrm{RC}$ ) that recognizes specifically each of the repeat sequences, and is sufficient to mediate function of the $d s x$ RE.

Tra and Tra2 form a specific complex with SF2/ASF or dSRp30 on the PRE

In contrast to the $d s \times \mathrm{RC}$, a similar but distinct heterotrimeric complex binds to the PRE. Cross-linking of proteins to the PRE was detected by the use of a single label within the middle of the PRE. We found that this single label was sufficient to assay the binding across the entire $\mathrm{PRE}$, and that placing labels at other positions along the PRE or uniformly labeling the PRE did not reveal any additional cross-linked proteins (data not shown; cf. Figs. $5 \mathrm{~A}$ and $6 \mathrm{~B}$ ). Thus, we were not able to identify binding sites along the PRE with the same resolution as was done for the repeat sequences.

The use of this PRE single-labeled RNA in cross-linking assays reveals the cross-linking of Tra2, Tra, and an SRp30 in both HeLa and Kc extracts (Fig. 5A). In contrast to the repeats, Tra cross-links much more strongly to the PRE than to the repeats, and in Kc extract dSRp30, but not RBP1, binds to the PRE. There is also a weak and somewhat variable association of the SR protein B52/ dSRp55 with the PRE single label, similar to the association of B52/dSRp55 with the uniformly labeled $d s x R E$ RNA. Because RBPl appears to act as the homolog to 9G8, the absence of RBP1 binding to the PRE suggests that the hSRp30 that cross-linked to the PRE is not 9G8.

Several previous studies have shown that SF2/ASF binds to purine-rich sequences (Sun et al. 1993) and, in particular, that SF2/ASF binds to the $d s x$ PRE (Lynch and Maniatis 1995). To determine whether the hSRp30 that cross-links to the PRE is SF2/ASF, we carried out immunoprecipitation studies with an anti-SF2 antibody. As shown in Figure 5B, anti-SF2 precipitates specifically a protein that cross-links strongly to the uniformly labeled $d s x$ RE in the presence of Tra and Tra2. Likewise, a protein that cross-links strongly to the PRE is also precipitated by anti-SF2. In contrast, no proteins that crosslink to either the $3^{\prime}$ half or the $5^{\prime}$ half of repeat 4 are precipitated by this antibody. These data confirm that 9G8, and not SF2/ASF, cross-links to the repeat sequences.

To further confirm the interaction of SF2/ASF with the PRE, we studied the binding of recombinant hSRp30s to the PRE in the presence or absence of Tra and Tra2 (Fig. 5C). In striking contrast to the binding of these proteins to the repeat sequences (Fig. 4A,B), SF2/ASF crosslinks to the PRE much more strongly and cooperatively with Tra and Tra2 than did either SC35 or 9G8. Thus, like the repeats, the PRE is also bound by a heterotrimeric complex containing Tra, Tra2, and an SR protein. However, the SR protein in this PRE-specific complex (ds $\mathrm{PCC})$ is SF2/ASF or dSRp30 as opposed to 9G8 or RBPl found in the $d s x$ RC.

Finally, we determined the roles Tra and Tra2 play independently in the formation of the $d s x \mathrm{PC}$. Previous studies suggested that Tra2 on its own was able to bind the PRE with some specificity, whereas isolated Tra did not recognize this sequence (Lynch and Maniatis 1995). Consistent with these results, we find that Tra2 stimulates the binding of Tra and either SF2/ASF or dSRp30 to the PRE (Fig. 5D) as well as being necessary for the weak interaction of B52/dSRp55 in Kc extract. Thus, Tra2 helps recruit these other factors to the PRE to form the dsxPC.

If we consider all of the proteins that cross-link to the repeats and PRE, we can account for all but one of the 

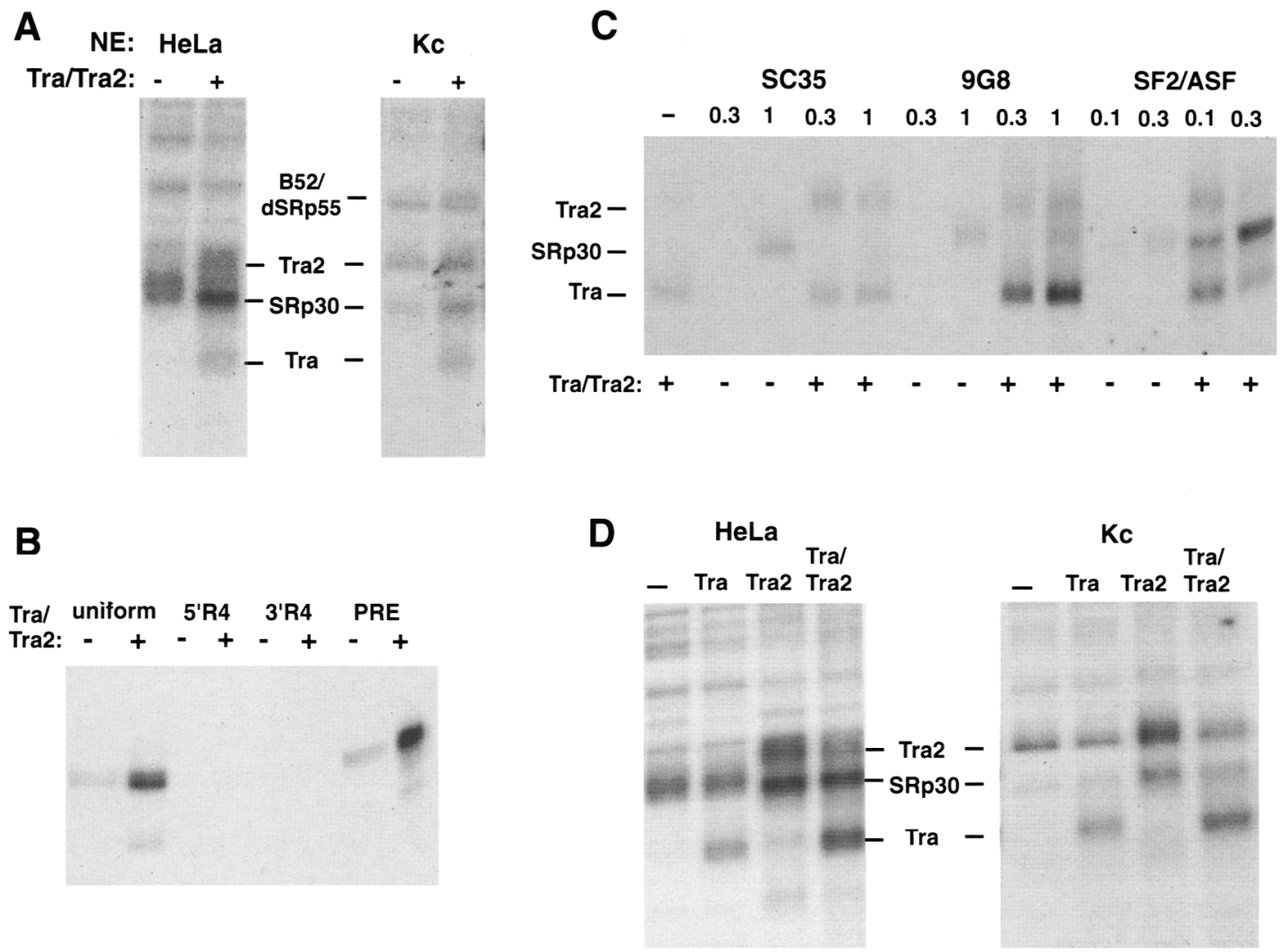

Figure 5. Site-specific cross-linking of proteins to the PRE identifies the association of SF2/ASF with the PRE. (A) Cross-linking to the singly labeled PRE (AAAGGACAAAG* GACAAA) within the context of the $d s x$ RE in the presence of HeLa or Kc extracts. (B) Immunoprecipitation of cross-linked reactions with anti-SF2. HeLa extract was cross-linked in the presence or absence of Tra/Tra2 to $d s x$ RE RNA that was labeled uniformly, or labeled in the $5^{\prime}$ or $3^{\prime}$ half of repeat 4, or within the PRE. After digestion with RNases the reactions were immunoprecipitated with a monoclonal antibody against SF2/ASF (see Materials and methods), and precipitated proteins were analyzed by SDS-PAGE gel. (C) Cross-linking of purified recombinant SC35, 9G8, and SF2/ASF in the presence and absence of Tra and Tra2 to the single PRE label. Units of SR proteins are given in microliters. All SR proteins used are at a concentration of approximately $100 \mathrm{ng} / \mu \mathrm{l}$. (D) Cross-linking to the PRE label when Tra alone, Tra2 alone, or Tra and Tra2 are added to HeLa or Kc extracts.

proteins that cross-link to the uniformly labeled $d s x \mathrm{RE}$, the SR protein hSRp20 (Fig. 1). To identify the site of hSRp20 cross-linking we introduced ${ }^{32} \mathrm{P}$ labels at various sites within the $d s x$ RE outside the repeats and PRE. We found that hSRp20 cross-links to an 8-nucleotide pyrimidine tract located between repeats 3 and 4 (data not shown). Studies are in progress to determine whether this interaction is functionally significant.

\section{The $\operatorname{dsx} R C$ and $\operatorname{dsx} P C$ form on target sequences in isolation}

Distinct heterotrimeric protein complexes are assembled on the repeat sequences and PRE within the intact $d s \times R E$. Thus, it is possible that the formation of these complexes depends on proteins that bind to other regions of the $d s x \mathrm{RE}$, or to the overall secondary structure of the RNA. Therefore, we carried out experiments to determine whether complexes of Tra, Tra2, and SR proteins can form on the isolated repeats or PRE. To examine complex formation on the repeat sequences we used uniformly labeled RNAs that contained either a single consensus repeat $(R \times 1)$ or a dimer of this repeat $(R \times 2)$. Both of these RNAs cross-link to Tra2 and 9G8 or RBP1, and weakly to Tra (Fig. 6A), the same proteins that cross-link to the repeat sequence within the intact $d s x$ RE. Additional studies demonstrated the affinity of the complex for the isolated repeats is approximately the same as that observed with the intact $d s x R E$ (data not shown). Thus, interrepeat cooperativity is not necessary for the stable association of Tra, Tra2, and SR proteins to the repeats.

We also carried out cross-linking assays using a uniformly labeled RNA that contained only the PRE sequence (Fig. 6B). This isolated PRE cross-linked to the same proteins, Tra, Tra2, and SF2/ASF or dSRp30, as did the PRE within the context of the $d s x$ RE. We did not detect cross-linking of B52/dSRp55 to the isolated PRE. However, the cross-linking of B52/dSRp55 to the singlelabeled PRE or to the uniformly labeled $d s x R E$ was also somewhat variable. Thus, neither neighboring sequences nor a particular structural conformation was necessary 
A

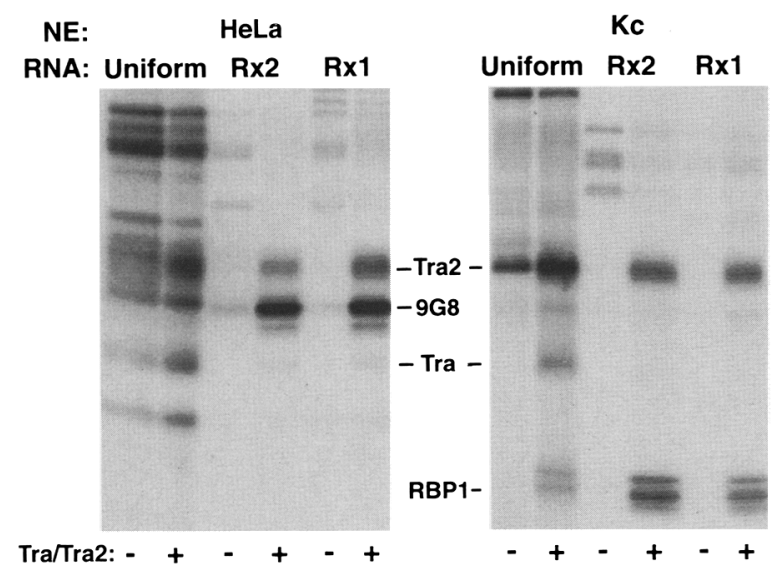

B

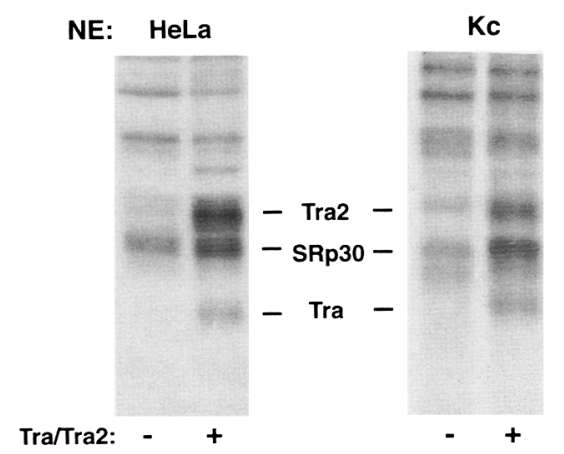

Figure 6. The $d s \times \mathrm{RC}$ or $d s x \mathrm{PC}$ are assembled on the corresponding elements in isolation. $(A)$ Cross-linking of proteins in HeLa or Kc extracts to $d s x$ RE RNA uniformly labeled with $\left[{ }^{32} \mathrm{P}\right] \mathrm{UTP}$, or $\mathrm{Rx} 2$ or $\mathrm{Rx} 1$ constructs uniformly labeled with $\left[{ }^{32} \mathrm{P}\right]$ ATP. The amount of each probe used was such that equal molar amounts of consensus repeat sequences are present in each reaction. Cross-linking is done with half as much nuclear extract and Tra/Tra2 as in other cross-linking experiments /see Materials and methods); thus conditions are in the linear range of detection. $(B)$ Cross-linking of HeLa or Kc extract in the presence and absence of Tra and Tra2 to the PRE uniformly labeled with $\left[{ }^{32}\right.$ P]ATP.

for the formation of specific heterotrimeric protein complexes on the repeat sequences or the PRE.

\section{Discussion}

We have shown that distinct SR protein complexes are assembled on the two types of regulatory elements in the $d s x$ splicing enhancer, and that the proteins in this complex are arranged in a specific $5^{\prime}$ to $3^{\prime}$ order (Fig. 7). Tra interacts only weakly with the middle of the repeat, but is essential for the cross-linking of $\operatorname{Tra} 2$ to the $3^{\prime}$ half of the repeat. The same set of proteins that cross-links to the consensus repeat 4 also cross-links to repeat 5 , although the $5^{\prime}$ end of repeat 5 deviates significantly from the consensus. Thus, it is likely that the same hetero- trimeric protein complexes are assembled on all of the repeat sequences.

We were unable to detect a specific order of protein binding on the PRE, most likely because of the scarcity of RNase cleavage sites within this element. However, it seems likely that, like the repeat sequences, the SR/Tra/ Tra2 proteins are arranged in a specific order on the PRE. Finally, site-specific labeling of the $d s x \mathrm{RE}$ outside the repeats or the PRE failed to detect any specific proteinRNA interactions, with the exception of the possible interaction of hSRp20 with the sequence between repeats 3 and 4 (data not shown). Taken together, these observations suggest strongly that the fully assembled $d s \times E C$ consists of seven tandemly arranged heterotrimeric protein-RNA complexes, six on the repeats and one on the PRE (Fig. 7).

The RNA-binding specificity of SR proteins is determined by a combination of protein-RNA and protein-protein interactions

Originally, the SR proteins were defined as a group by their copurification, and individually by their ability to activate splicing in SR protein-deficient S100 extracts (Fu and Maniatis 1992; Mayeda et al. 1992; Zahler et al. 1992). Because all of the SR proteins tested showed activity in this assay, it appeared that individual SR proteins are functionally redundant. However, subsequent studies demonstrated that certain SR proteins could promote splicing preferentially (Zahler et al. 1993; Wang

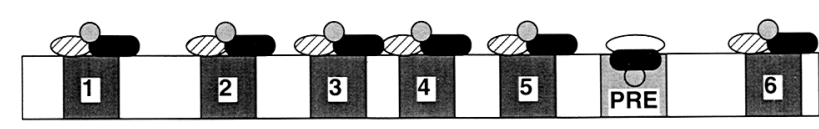

Repeats:
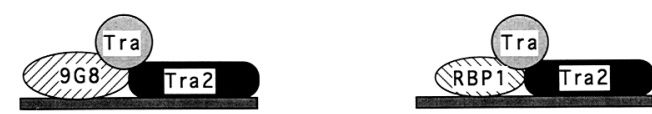

PRE:
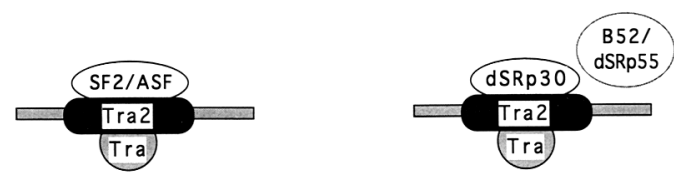

Human

Drosophila

Figure 7. Model of the protein-protein and protein-RNA interactions involved in the formation of the $d s x E C$. Data presented in this study are summarized in the complexes bound to the repeats or the PRE. Within the full $d s x$ RE we have shown that the same repeat-specific complex binds to repeats 4 and 5 . Given the sequence similarity between repeats 1,2 , and 3 and repeats 4 and 5 , we can extrapolate that the same complex binds to repeats 1 through 5 . Repeat 6 is the most dissimilar of the repeat sequences; however, we hypothesize, that the same repeat-specific complex binds to this sequence as to the other repeats. 
and Manley 1995) and commitment complex formation (Fu 1993) with different splicing substrates. In addition, distinct SR proteins were shown to associate with different constitutive (Lavigueur et al. 1993; Sun et al. 1993; Ramchatesingh et al. 1995) and regulated (Tian and Maniatis 1993) splicing enhancers. These associations could be correlated with the ability of these proteins to promote splicing in vitro. Similarly, multiple base substitutions in a purine-rich splicing enhancer had little effect on the activity of the enhancer, but did result in a dramatic switch in the SR proteins that interact specifically with the sequence (Staknis and Reed 1994). Thus, individual SR proteins can associate specifically with different pre-mRNAs, presumably attributable to amino acid sequence differences in their RNA-binding domains.

As expected from such a proposal, when the RNAbinding domains of SC35, SF2/ASF, and RBP1 were used to identify RNA recognition sequences by in vitro selection (SELEX), each protein selected a different RNA sequence (Heinrichs and Baker 1995; Tacke and Manley 1995). In the case of SC35 and SF2/ASF these differences in binding specificity could be correlated with in vitro function (Tacke and Manley 1995).

The situation in vivo is likely to be more complex because of the possibility of multiple protein-protein interactions through the SR domains of these proteins. Although different SR domains display relatively little specificity in protein-protein interaction assays $(\mathrm{Wu}$ and Maniatis 1993; Amrein et al. 1994; Kohtz et al. 1994), the results presented here show that highly specific protein-RNA complexes can be formed through a combination of protein-RNA and protein-protein interactions. Moreover, the RNA sequence recognized by the complex is determined by the particular set of proteins assembled. Specifically, we show that Tra and Tra2 can associate with entirely different RNA sequences, the repeats, or the PRE by associating with different SR proteins. Furthermore, neither Tra2 nor 9G8 or RBP1 binds individually with high affinity to the UCAACA sequence within the repeats. However, all of these proteins can interact strongly with this sequence, depending on whether the position of this sequence is at the $5^{\prime}$ or $3^{\prime}$ end of the repeat. Thus, the specificity of SR proteinRNA interactions is determined by a combination of protein-protein and protein-RNA interactions.

Three different assays have been used to study specific protein-RNA interactions in the $d s x \mathrm{EC}$, and the results are consistent. First, we show here that Tra and Tra2 recruit specifically the SR proteins 9G8 and SF2/ASF to the repeats and the PRE, respectively, as assayed by UVcross-linking of extracts to singly-labeled $d s x$ RE probes. This is a highly sensitive assay, as no exogenous SR proteins are added, and 9G8 and SF2/ASF are selected from the total population of SR proteins in the nuclear extract.

Second, the functional significance of 9G8 recruitment was demonstrated in both the commitment complex and $\$ 100$ reconstitution assays, where 9G8 was the most effective of the SR proteins tested. Although SC35 can function in this assay, higher levels of protein are required (Tian and Maniatis 1993). Similarly, SC35, but not SF2/ASF, can complement splicing activity in a 9G8depleted extract (Cavaloc et al. 1994), indicating that there is some functional redundancy between 9G8 and SC35.

The functional significance of the recruitment of SF2/ ASF to the PRE has been more difficult to demonstrate as this protein is not active in the commitment complex assay (Fig. 4; Tian and Maniatis 1993). However, we note that the binding of a protein to the $d s x$ PRE may not be sufficient to commit the pre-mRNA to the female-specific pathway of splicing. One reason for this may be that only one copy of the PRE is present in the $d s x R E$ and that this PRE is insufficient to stimulate the use of the female-specific 3' splice site (Lynch and Maniatis 1995). This may explain why SF2/ASF does not function in the commitment complex assay (Tian and Maniatis 1993).

We also find that B52/dSRp55 is weakly recruited to the PRE by Tra and Tra2. In contrast to SF2/ASF, this protein has been shown to function with Tra and Tra2 in a commitment complex assay (Tian and Maniatis 1993). One explanation for this apparent contradiction is that, unlike SF2/ASF, B52/dSRp55 can bind to the repeats in vitro in the absence of other SR proteins (Lynch and Maniatis 1995). Thus, although neither SC35 nor B52/ dSRp55 are recruited to the repeats in nuclear extract, both bind to the repeats as purified recombinant proteins, and both can function in the commitment complex assay.

Genetic studies have shown that a dominant-negative mutant of B52/dSRp55 over wild type decreased femalespecific splicing of $d s x$ pre-mRNA splicing in a background of reduced levels of Tra and Tra2 (Peng and Mount 1995). However, when a B52/dSRp55 null over wild type was examined in the same genetic background no effect on $d s x$ splicing was observed. Homozygous B52/dSRp55 null mutations are lethal (Ring and Lis 1994; Peng and Mount 1995). Thus, genetic studies of B52/dSRp55 cannot determine unambiguously whether this protein plays an essential role in female-specific splicing of $d s x$ pre-mRNA in the fly.

A third assay used to study the $d s \times \mathrm{EC}$ was filter binding with radiolabeled RNA and purified recombinant proteins (Lynch and Maniatis 1995). These studies showed that recombinant SC35 can be recruited to the intact $d s \times \mathrm{RE}$ by Tra and Tra2, but recombinant 9G8 was not available at the time. In this paper we show that recombinant 9G8 is cross-linked efficiently to the $d s x \mathrm{RE}$ in the presence of Tra2 (Fig. 3A). In contrast, similar amounts of recombinant $\mathrm{SC} 35$ are not recruited to the $d s x$ RE by Tra and Tra2. The overall results obtained with these three assays strongly support the hypothesis that specific SR proteins are normally recruited to the $d s x E C$ by Tra and Tra2, but other SR proteins can bind and function at higher concentrations.

\section{$9 G 8$ and RBP1 may be functional homologs}

The cross-linking results presented here strongly suggest that Drosophila RBP1 and human 9G8 are functional homologs. Although we have not yet been able to test 
RBP1 in splicing assays, we were able to show that RBP1 functions similarly to $9 \mathrm{G} 8$ in the formation of the $d s x$ RCs. Previously, RBPl was thought to be the Drosophila equivalent of human SRp20 or SF2/ASF (Kim et al. 1992). However, in this study we show that RBPl binds to the same sites as 9G8, and does not interact with sites bound by either SF2/ASF or hSRp20. Although RBP1 and hSRp20 are similar in molecular mass and contain similar RNA-binding domains (RRMs), 9G8 also shares extensive homology with RBPl within the RRMs of the respective proteins. The RRMs of RBP1 and either 9G8 or SRp 20 are $71 \%$ identical and $83 \%$ similar, whereas SF2/ASF and RBP1 share only $53 \%$ sequence identity (71\% similarity) between their RRMs /Cavaloc et al. 1994). At present, we do not know why 9G8 is favored over hSRp20 in the binding of the repeats; presumably this is a consequence of differences in the interactions of these proteins with Tra and Tra2.

As mentioned previously, Heinrichs and Baker (1995) determined potential binding sites for RBP1 within the $d s x \mathrm{RE}$ by comparison with consensus-binding sequences that were identified using purified RBP1 in SELEX experiments. Therefore, their study was designed to identify sites that bound to RBP1 alone. In contrast, we find that under the conditions used here RBP1 does not bind to the $d s x \mathrm{RE}$ in the absence of Tra and Tra2. Furthermore, we do not detect any binding of RBPl, in either the presence or absence of Tra and Tra2, to one of the proposed RBP1 sites (B3; Heinrichs and Baker 1995) in which we placed a single label (data not shown). Instead we find that Tra2, and sometimes Tra, is required for the binding of RBPl to the $d s x \mathrm{RE}$, and that under these conditions RBP1 binds to the $5^{\prime}$ half of the repeat. Although, with the exception of the first repeat, Heinrichs and Baker (1995) did not generally identify the sequences at the 5 ' half of the repeats as RBP1-binding sites, these sequences differ at only one or two positions from one of the consensus sequences identified in that study. Thus, the site to which RBP1 binds within the repeat-specific complex may be a sequence for which RBP1 alone has some weak affinity; however, Tra2 and Tra are required for optimal recognition of the site by RBP1.

Evidence for a function of RBPl in sex determination was provided by genetic studies that showed that deletion of the chromosomal region in which the $r b p 1$ gene is located leads to intersex phenotype when the levels of Tra and Tra2 are reduced (Scott 1987). Although additional studies are required, the results of these genetic experiments, and the fact that RBPl is recruited selectively to the $d s x$ repeats in vitro, strongly suggest that this SR protein plays a central role in $d s x$ alternative splicing.

The heterotrimeric SR protein complexes assemble on isolated repeat and PRE sequences

We have shown that the SR/Tra/Tra2 protein complexes form not only on the repeats and the PRE in the context of the intact $d s x \mathrm{RE}$, but also on these sequences in isolation. In addition, the affinity and stability of these pro-
tein-RNA complexes are context independent. Thus, neither the formation nor the stability of the heterotrimeric protein complexes requires cooperativity between the repeats or between the repeats and the PRE.

Maximal levels of Tra- and Tra2-dependent splicing require multiple repeats and the PRE (Hoshijima et al. 1991; Tian and Maniatis 1992; Lynch and Maniatis 1995). In addition, both the rates and efficiency of splicing increase in relation to the number of repeats present in the $d_{s x} \mathrm{RE}$ (K.J. Hertel and T. Maniatis, in prep.). Recent studies have demonstrated that Tra, Tra2, and several SR proteins can interact with the $35-\mathrm{kD}$ subunit of $\mathrm{U} 2 \mathrm{AF}\left(\mathrm{U}_{2} \mathrm{AF}^{35}\right)$ (Wu and Maniatis 1993), that the $d s \times \mathrm{EC}$ acts by recruiting the general splicing factor $U 2 \mathrm{AF}^{65}$ to the weak female-specific $3^{\prime}$ splice site, and that $\mathrm{U} \mathrm{AF}^{35}$ mediates this interaction (Zuo and Maniatis 1996). Thus, the complexes formed on the multiple repeats and the PRE appear to increase the probability of a productive interaction between the enhancer complex and $\mathrm{U} 2 \mathrm{AF}^{35}$ to facilitate use of the female-specific 3 ' splice site.

\section{Tra mediates protein-protein and protein-RNA} interactions

The sex-specific regulation of $d s x$ in vivo is determined solely by the presence or absence of Tra, the only femalespecific component of the $d s x$ regulatory system. Surprisingly, however, Tra contains no known RNA-binding motif. Furthermore, although Tra does cross-link with high efficiency to the PRE, this sequence is not sufficient, and also not necessary, for the female-specific splicing of $d s x$. In contrast, Tra cross-links only weakly to the repeat sequences. However, despite its weak interactions with the repeat sequences themselves, Tra is necessary for the optimal association of Tra2 with the repeats. Thus, Tra does not appear to regulate splicing by directly interacting with the RNA; instead, it functions through interactions with Tra2, and perhaps also with the SR proteins that are critical for proper assembly of the $d s x \mathrm{EC}$. This function is reminiscent of that of the $35-\mathrm{kD}$ subunit of the splicing factor U2AF (U2AF ${ }^{35}$ ). Similar to Tra, $\mathrm{U}_{2} \mathrm{AF}^{35}$ contains an SR domain, but lacks an RRM (Zhang et al. 1992). Recently, U2AF ${ }^{35}$ was shown to be essential for both constitutive and enhancer-dependent splicing in vitro (Zuo and Maniatis 1996), and probably functions in both processes by mediating interactions between SR proteins containing an RRM. Thus, both Tra and $\mathrm{U}_{2} \mathrm{AF}^{35}$ may function by facilitating the cooperative binding of proteins to specific RNA sequences.

\section{Materials and methods}

\section{RNA}

The $d s x$ RE RNA was transcribed in vitro from a construct in which repeats 1-6, flanked by a PCR-generated EcoRI site and an FspI site, were subcloned downstream of the $\mathrm{T} 7$ promoter in pSP72. The $d s x$ splicing construct used was D1 (Tian and Maniatis 1992|, which lacks only repeat 6 and is more stable in HeLa nuclear extract than constructs containing repeat 6 /data 
not shown). The $\beta$-globin splicing construct is as described in Reed et al. (1988). Both the $d s x \mathrm{RE}$ and the splicing constructs were uniformly labeled with $\left[{ }^{32} \mathrm{P}\right] \mathrm{UTP}$. The Rx2 RNA was transcribed from a construct made by subcloning the sequence 5'-GGATCCGTCTTCAATCAACATACGCGAGATCT-3' cut with $B a m I$ and $B g l I I$ into the $B g I I I$ site of $\mathrm{pSP} 72$, such that dimers of the sequence were incorporated into the plasmid. Rxl was made by cloning the fragment $5^{\prime}$-CGTCTTCAATCAACATACGCGTCTCT'TCAATCAACACGGTAC-3' into the KpnI site of pBluescript SK + (Stratagene) and linearizing with MluI for in vitro transcription. The isolated PRE was synthesized from a construct in which the region spanning exons 3 and 4 was removed from $d s x$ PRE (Lynch and Maniatis 1995). Rx2, $\mathrm{Rxl}$, and the isolated PRE were all uniformly labeled with $\left[{ }^{32} \mathrm{P} \mid \mathrm{ATP}\right.$

Site-specific single-labeled RNAs were synthesized in most cases by ligating a 5' half RNA to a 5' end-labeled 3' half RNA using the method of Moore and Sharp (1992). Templates for the synthesis of the $5^{\prime}$ and $3^{\prime}$ RNAs were generated by PCR from the $d s \times \mathrm{RE}$ construct, so that the resulting single label is in the context of the entire $d s x$ RE. The $5^{\prime}$ half templates began with the $\mathrm{T} 7$ promoter from pSP72 and ended precisely at the nucleotide immediately upstream from the one to be labeled. For the 3 ' half templates, the T7 promoter was placed immediately upstream from the nucleotide to be labeled by using PCR primers that contained the $\mathrm{T} 7$ promoter. Both $5^{\prime}$ and $3^{\prime}$ half RNAs were then transcribed in vitro from these PCR-generated templates. After synthesis of RNA, the 3' half RNA was treated with calf intestine phosphatase for $1 \mathrm{hr}$ at $50^{\circ} \mathrm{C}$, and then $5^{\prime}$ end-labeled by a kinase reaction. The two half RNAs were then ligated in a reaction that contained $1 \mu \mathrm{M}$ each of the RNAs and of a DNA bridge, and 60-70 U/ $\mu$ l T4 DNA ligase (Moore and Sharp 1992).

To place a single label in the $5^{\prime}$ half of repeat 4 we needed to label a uracil residue, which is not used efficiently to initiate $\mathrm{T} 7$ transcripts. Therefore, this single-labeled RNA was made by a three-way ligation. An RNA oligonucleotide, $5^{\prime}$-UCAAUCAACAAUCC-3', was synthesized chemically, kinased, and ligated to two flanking RNAs that contained the remaining $d s x$ RE sequences.

\section{Recombinant proteins}

Recombinant baculoviruses expressing Tra and Tra2, and the purification of Tra and Tra2 were as described in Tian and Maniatis (1992). Recombinant viruses expressing SF2/ASF and SC35, and the purification of these proteins were as described in Tian and Maniatis (1993). Recombinant 9G8 was a gift of $R$. Gattoni (CNRS, Strasbourg, France) and J. Stevenin.

\section{In vitro splicing reactions}

In vitro splicing reactions were carried out as described in Tian and Maniatis (1992). Reactions were in a total volume of $25 \mu 1$ and contained $25 \%$ nuclear extract or $\mathrm{S} 100$ extract. Commitment complex assays were performed as described in Tian and Maniatis (1993).

\section{UV cross-linking}

Standard UV cross-linking experiments were done by incubating $20 \%$ nuclear extract with $\sim 1 \mathrm{ng}$ of RNA in either the presence or absence of $60 \mathrm{ng}$ each of recombinant Tra and Tra 2 for $30 \mathrm{~min}$ at $30^{\circ} \mathrm{C}$. The sole exception to these conditions was the experiment shown in Figure 6A in which only half as much nuclear extract, Tra, and Tra2 were used. Binding reactions also contained $145 \mathrm{mM} \mathrm{KCl}, 0.5 \mathrm{mg} / \mathrm{ml}$ of yeast tRNA, $20 \mu \mathrm{g} / \mathrm{ml}$ of BSA, $3.2 \mathrm{mM} \mathrm{MgCl}_{2}, 1 \mathrm{~mm}$ ATP, and $20 \mathrm{~mm}$ creatine phosphate in a total volume of $10 \mu \mathrm{l}$. After binding, the reactions were placed on ice and cross-linked with $254 \mathrm{~nm}$ UV light for $15 \mathrm{~min}$. RNase A $(20 \mu \mathrm{g})$ and Tl (20 units) were then added and the reactions were incubated at $37^{\circ} \mathrm{C}$ for $20 \mathrm{~min}$. RNase digestion was stopped by the addition of Laemmli buffer, and then samples were boiled for $5 \mathrm{~min}$ and loaded on a $12.5 \%$ SDS-PAGE gel.

For immunoprecipitation of cross-linked reactions with antiSF2 antibody, $250 \mu 1$ of $2 \times$ RIPA buffer and $235 \mu$ lof anti-SF2 cell supernatant were added to samples after RNase digestion. After incubation at $4^{\circ} \mathrm{C}$ for $1 \mathrm{hr}, 20 \mu \mathrm{l}$ of protein G-Sepharose beads were added to the samples and incubation was continued for another hour. The samples were then spun to precipitate the beads, and the beads were washed four times with $200 \mu l$ of $1 \times$ RIPA buffer at $4^{\circ} \mathrm{C}$. Finally, the beads were resuspended in $20 \mu \mathrm{l}$ of Laemmli buffer, boiled for $5 \mathrm{~min}$ and loaded on a $12.5 \%$ SDS-PAGE gel.

The antibodies used for Western blots (data not shown) are as follows: anti-SF2 (Sun et al. 1993) and anti-Tra2 (Hedley and Maniatis 1991), which were both also used for immunoprecipitations, are mouse monoclonal antibodies. Anti-9G8, antiSC35 (both kindly provided by J. Stevenin), and anti-RBPl (Kim et al. 1992; kindly provided by V. Heinrichs and B. Baker, Stanford University, Palo Alto, CA/ are rabbit polyclonal antibodies. Of these three antibodies only anti-RBPl was effective for use in immunoprecipitation experiments.

\section{Acknowledgments}

We are grateful to Renate Gattoni and James Stevenin for the gift of recombinant $9 \mathrm{G} 8$, and anti-9G8 and anti-SC35 antibodies, Volker Heinrichs and Bruce Baker for the gift of anti-RBP1 antibodies, Akila Mayeda and Adrian Krainer for the gift of antiSF2 antibodies and the S100 extract, and Jeremiah Hagler for the $\mathrm{Kc}$ cell extract. We also thank members of the Maniatis laboratory for critical comments on the manuscript.

The publication costs of this article were defrayed in part by payment of page charges. This article must therefore be hereby marked "advertisement" in accordance with 18 USC section 1734 solely to indicate this fact.

\section{References}

Amrein, H., M. Gorman, and R. Nöthiger. 1988. The sex-determining gene tra-2 of Drosophila encodes a putative RNA binding protein. Cell 55: 1025-1035.

Amrein, H., M.L. Hedley, and T. Maniatis. 1994. The role of specific protein-RNA and protein-protein interactions in positive and negative control of pre-mRNA splicing by Transformer 2. Cell 76: 735-746.

Baker, B.S. 1989. Sex in flies: The splice of life. Nature 340: 521-524.

Bennett, M., S. Pinol-Roma, D. Staknis, G. Dreyfuss, and R. Reed. 1992. Differential binding of heterogeneous nuclear ribonucleoproteins to mRNA precursors prior to spliceosome assembly in vitro. Mol. Cell. Biol. 12: 3165-3175.

Boggs, R.T., P. Gregor, S. Idriss, J.M. Belote, and M. McKeown. 1987. Regulation of sexual differentiation in D. melanogaster via alternative splicing of RNA from the transformer gene. Cell 50: 739-747.

Burtis, K.C. and B.S. Baker. 1989. Drosophila doublesex gene 
controls somatic sexual differentiation by producing alternative spliced mRNAs encoding related sex-specific polypeptides. Cell 56: 997-1010.

Cavaloc, Y., M. Popielarz, J.P. Fuchs, R. Gattoni, and J. Stevenin. 1994. Characterization and cloning of the human splicing factor 9G8: A novel $35 \mathrm{kDa}$ factor of the serine/arginine protein family. $E M B O /$. 13: 2639-2649.

Coschigano, K.T. and P.C. Wensink. 1993. Sex-specific transcriptional regulation by the male and female doublesex proteins of Drosophila. Genes \& Dev. 7: 42-54.

Fu, X.-D. 1993. Specific commitment of different pre-mRNAs to splicing by single SR proteins. Nature 343: 82-85.

. 1995. The superfamily of arginine/serine-rich splicing factors. RNA 1: 663-680.

Fu, X.-D. and T. Maniatis. 1992. Isolation of a complementary DNA that encodes the mammalian splicing factor SC35. Science 256: 535-538.

Ge, H., P. Zuo, and J.L. Manley. 1991. Primary structure of the human splicing factor ASF reveals similarities with Drosophila regulators. Cell 66: 373-382.

Hedley, M.L. and T. Maniatis. 1991. Sex-specific splicing and polyadenylation of $d s x$ pre-mRNA requires a sequence that binds specifically to tra-2 protein in vitro. Cell 65: 579-586.

Hedley, M.L., H. Amrein, and T. Maniatis. 1995. An amino acid sequence motif sufficient for subnuclear localization of an arginine/serine-rich splicing factor. Proc. Natl. Acad. Sci. 92: 11524-11528

Heinrichs, V. and B.S. Baker. 1995. The Drosophila SR protein $\mathrm{RBPl}$ contributes to the regulation of doublesex alternative splicing by recognizing RBP1 RNA target sequences. $E M B O$ J. 14: 3987-4000.

Hoshijima, K., K. Inoue, I. Higuchi, H. Sakamoto, and Y. Shimura. 1991. Control of doublesex alternative splicing by transformer and transformer-2 in Drosophila. Science 252: 833-836.

Kim, Y.J., P. Zuo, J.L. Manley, and B.S. Baker. 1992. The Drosophila RNA-binding protein RBPl is localized to transcriptionally active sites of chromosomes and shows a functional similarity to human splicing factor ASF/SF2. Genes \& Dev. 6: 2569-2579.

Kohtz, J.D., S.F. Jamison, C.L. Will, P. Zuo, R. Lührmann, M. Garcia-Blanco, and J.L. Manley. 1994. Protein-protein interactions and 5 '-splice site recognition in mammalian mRNA precursors. Nature 368: 119-124.

Krainer, A.R., A. Mayeda, K. Kozak, and G. Binns. 1991. Functional expression of clones of human splicing factor SF2: Homology to RNA binding proteins, U1 70K, and Drosophila splicing regulators. Cell 66: 383-394.

Lavigueur, A., H. La Branche, A.R. Kornblihtt, and B. Chabot. 1993. A splicing enhancer in the human fibronectin alternative ED1 exon interacts with SR proteins and stimulates U2 snRNP binding. Genes \& Dev. 7: 2405-2417.

Lynch, K.W. and T. Maniatis. 1995. Synergistic interaction between two distinct elements of a regulated splicing enhancer. Genes \& Dev. 9: 284-293.

Maniatis, T. 1991. Mechanisms of alternative pre-mRNA splicing. Science 251: 33-34.

Mayeda, A., A.M. Zahler, A.R. Krainer, and M.B. Roth. 1992. Two members of a conserved family of nuclear phosphoproteins are involved in pre-mRNA splicing. Proc. Natl. Acad. Sci. 89: 1301-1304.

McKeown, M., J.M. Belote, and R.T. Boggs. 1988. Ectopic expression of the female transformer gene product leads to female differentiation of chromosomally male Drosophila. Cell 53: 887-895.

Moore, M. and P.A. Sharp. 1992. Site-specific modification of
pre-mRNA: The 2'-hydroxyl group at the splice site. Science 256: 992-996.

Nagoshi, R.N. and B.S. Baker. 1990. Regulation of sex-specific RNA splicing at the Drosophila doublesex gene: cis-acting mutations in exon sequences alter sex-specific RNA splicing patterns. Genes \& Dev. 4: 89-97.

Nagoshi, R.N., M. McKeown, K.C. Burtis, J.M. Belote, and B.S. Baker. 1988. The control of alternative splicing at genes regulating sexual differentiation in D. melanogaster. Cell 53: 229-236.

Peng, X. and S.M. Mount. 1995. Genetic enhancement of RNAprocessing defects by a dominant mutation in B52, the Drosophila gene for an SR protein splicing factor. Mol. Cell. Biol. 15: 6273-6282.

Ramchatesingh, J., A.M. Zahler, K.M. Neugebauer, M.B. Roth, and T.A. Cooper. 1995. A subset of SR proteins activates splicing of the cardiac troponin $\mathrm{T}$ alternative exon by directing interactions with an exonic enhancer. Mol. Cell. Biol. 15: 4898-4907.

Reed, R., J. Griffith, and T. Maniatis. 1988. Purification and visualization of native spliceosomes. Cell 53: 949-961.

Ring, H.Z. and J.T. Lis. 1994. The SR protein B52/SRp55 is essential for Drosophila development. Mol. Cell. Biol. 14: 7499-7506.

Rio, D. 1992. RNA binding proteins, splice site selection and alternative pre-mRNA splicing. Gene Expr. 2: 1-5.

Roth, M.B., A.M. Zahler, and J.A. Stolk. 1991. A conserved family of nuclear phosphoproteins localized to sites of polymerase II transcription. J. Cell. Biol. 115: 587-596.

Ryner, L.C. and B.S. Baker. 1991. Regulation of doublesex premRNA processing occurs by 3 '-splice site activation. Genes \& Dev. 5: 2071-2085.

Scott, T.N. 1987. "Identification and characterization of a region of the $\mathrm{X}$ chromosome of Drosophila melanogaster involved in sex determination." $\mathrm{PhD}$ thesis. University of California, San Diego, CA.

Staknis, D. and R. Reed. 1994. SR proteins promote the first specific recognition of pre-mRNA and are present together with the Ul small nuclear ribonucleoprotein particle in a general splicing enhancer complex. Mol. Cell. Biol. 14: $7670-7682$.

Steinmann-Zwicky, M., H. Amrein, and R. Nöthiger. 1990. Genetic control of sex determination in Drosophila. Adv Genet. 27: 189-237.

Sun, Q., A. Mayeda, R.K. Hampson, A.R. Krainer, and F.M. Rottman. 1993. General splicing factor SF2/ASF promotes alter native splicing by binding to an exonic splicing enhancer. Genes \& Dev. 7: 2598-2608.

Tacke, R. and J.L. Manley. 1995. The human splicing factors ASF/SF2 and SC35 possess different, functionally significant, RNA binding specificities. EMBO I. 14: 3540-3551.

Tian, M. and T. Maniatis. 1992. Positive control of pre-mRNA splicing in vitro. Science 256: $237-240$

. 1993. A splicing enhancer complex controls alternative splicing of doublesex pre-mRNA. Cell 74: 105-114.

- 1994. A splicing enhancer exhibits both constitutive and regulated activities. Genes \& Dev. 8: 1703-1712.

Wang, J. and J.L. Manley. 1995. Overexpression of the SR proteins ASF/SF2 and SC35 influences alternative splicing in vivo in diverse ways. RNA 1: 335-346.

Wu, J.Y. and T. Maniatis. 1993. Specific interactions between proteins implicated in splice site selection and regulated alternative splicing. Cell 75: 1061-1070.

Zahler, A.M., W.S. Lane, J.A. Stolk, and M.B. Roth. 1992. SR proteins: A conserved family of pre-mRNA splicing factors. Genes \& Dev. 6: 837-847. 
Zahler, A.M., K.M. Neugebauer, W.S. Lane, and M.B. Roth. 1993. Distinct functions of SR proteins in alternative premRNA splicing. Science 260: 219-222.

Zhang, M., P.D. Zamore, M. Carmo-Fonseca, A.I. Lamond, and M.R. Green. 1992. Cloning and intracellular localization of the U2 small nuclear ribonucleoprotein auxiliary factor small subunit. Proc. Natl. Acad. Sci. 89: 8769-8773.

Zuo, P. and T. Maniatis. 1996. The splicing factor U2AF ${ }^{35}$ mediates critical protein-protein interactions in constitutive and enhancer-dependent splicing. Genes \& Dev. 10: 13561368 . 


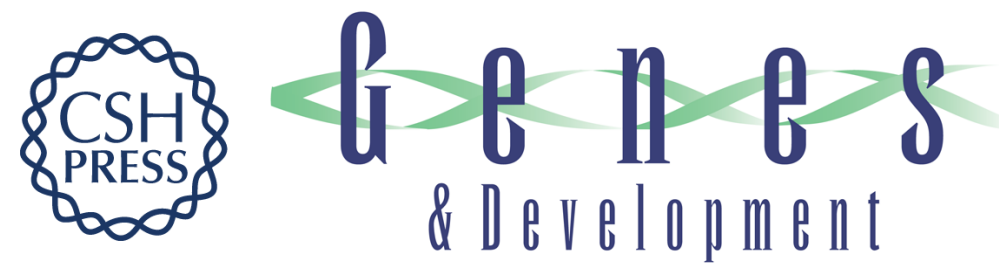

\section{Assembly of specific SR protein complexes on distinct regulatory elements of the Drosophila doublesex splicing enhancer.}

$\mathrm{K}$ W Lynch and T Maniatis

Genes Dev. 1996, 10:

Access the most recent version at doi:10.1101/gad.10.16.2089

References This article cites 47 articles, 25 of which can be accessed free at:

http://genesdev.cshlp.org/content/10/16/2089.full.html\#ref-list-1

License

Email Alerting

Service

Receive free email alerts when new articles cite this article - sign up in the box at the top right corner of the article or click here.

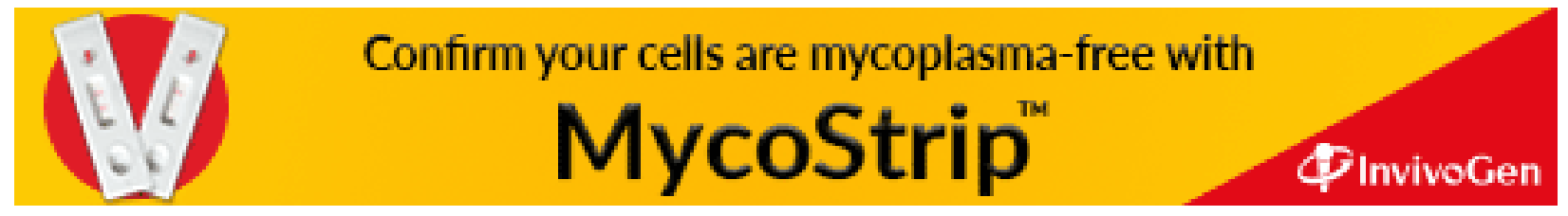

